\title{
Long noncoding RNA cancer susceptibility candidate 9 promotes doxorubicin-resistant breast cancer by binding to enhancer of zeste homolog 2
}

\author{
BAOHONG JIANG ${ }^{1}$, YUEHUA $\mathrm{LI}^{2}$, XIAOFEI QU ${ }^{2}$, HONGBO ZHU $^{2}$, YERU TAN $^{2}$, \\ QUN FAN $^{2}$, YILING JIANG ${ }^{2}$, MINGCHU LIAO $^{2}$ and XIAOPING WU ${ }^{2}$ \\ Departments of ${ }^{1}$ Pharmacy and ${ }^{2}$ Medical Oncology, The First Affiliated Hospital of \\ University of South China, Hengyang, Hunan 421001, P.R. China
}

Received July 10, 2017; Accepted July 24, 2018

DOI: $10.3892 /$ ijmm.2018.3812

\begin{abstract}
The present study aimed to investigate the effect of the long noncoding RNA cancer susceptibility candidate 9 (CASC9) on doxorubicin (DOX)-resistant breast cancer and to reveal the potential underlying mechanisms. The expression of CASC9 in breast cancer tissues and cell lines, in addition to drug-resistant breast cancer cells (MCF-7/DOX), was detected by reverse transcription-quantitative polymerase chain reaction. Subsequently, MCF-7/DOX cells were transfected with the silencing vector pS-CASC9, containing enhancer of zeste homolog 2 (EZH2), multidrug resistance protein 1 (MDR1) or control small interfering (si)RNAs. The viability, apoptosis, migration and invasion of the transfected cells were assessed via an MTT assay, flow cytometry and a Transwell assay, respectively. The expression levels of apoptosis-associated proteins (apoptosis regulator $\mathrm{Bcl}-2$, apoptosis regulator BAX, caspase-3 and caspase-9) were determined by western blotting. An RNA pull-down assay was performed to identify CASC9-binding candidates. In addition, the expression levels of the MDR1 gene and its encoded protein, P-glycoprotein, were detected. CASC 9 expression was upregulated in breast cancer tissues and cell lines, and drug-resistant breast cancer cells. CASC9 knockdown significantly inhibited the growth and metastasis of drug-resistant breast cancer cells, and decreased the half-maximal inhibitory concentration DOX in MCF-7/DOX cells. The RNA pull-down assay revealed that CASC9 engaged EZH2; EZH2 siRNA significantly inhibited the cell growth, metastasis and chemoresistance of MCF-7/DOX cells. Additionally, EZH2 may regulate the
\end{abstract}

Correspondence to: Professor Yuehua Li, Department of Medical Oncology, The First Affiliated Hospital of University of South China, 69 Chuanshan Road, Hengyang, Hunan 421001, P.R. China E-mail: liyuehua05@126.com

Key words: breast cancer, drug-resistance, cancer susceptibility candidate 9 , enhancer of zeste homolog 2 , multidrug resistance protein 1
MDR1 gene. The present study demonstrated the oncogenic role of CASC9 in drug-resistant breast cancer by binding to EZH2 and regulating the MDR1 gene. Modulation of CASC9 expression may be a promising target in the therapy of breast cancer and drug-resistant breast cancer.

\section{Introduction}

Breast cancer is the most common form of female cancer worldwide (1). It is a phenotypically and genetically complex disease; the progression and development of breast cancer has been associated with many factors (2). According to the report of the World Health Organization, there were 1.68 million cases of and 522,000 mortalities due to breast cancer in 2012 (3). Chemotherapy is the principal therapeutic method for patients with breast cancer (4); doxorubicin (DOX) is one of the most effective chemotherapeutic drugs for the treatment of breast cancer, which may induce regression of metastatic breast cancer (5). However, the effectiveness of DOX is hindered by the capacity of tumor cells to develop resistance to anticancer therapies (6). Therefore, understanding the mechanism of cancer-specific drug resistance may help to inhibit or overcome this resistance in breast cancer (7).

The causes of cancer-specific drug resistance have been associated with drug-induced karyotypic alterations, random drug-induced mutational events and non-mutational alterations of gene function (8-10). Additionally, studies have reported another mechanism of non-mutational regulation of gene function mediated by non-protein-coding RNAs (ncRNAs) $(11,12)$. Long (1)ncRNAs are RNA molecules with >200 nucleotides (13). Extensive studies have suggested that lncRNAs serve key regulatory roles in numerous biological processes, which are increasingly recognized as biomarkers of numerous types of cancer, including breast cancer $(14,15)$. IncRNA cancer susceptibility candidate 9 (CASC9), located in the human chromosome $8 \mathrm{q} 21.11$, was originally reported to be abnormally expressed in esophageal squamous cell carcinoma (16). Recent studies indicated that CASC9 is associated with a variety of cancer types, including gastric cancer and nasopharyngeal carcinoma $(17,18)$. The role of CASC9 in breast cancer and drug-resistant breast cancer remains to be examined. 
To the best of our knowledge, the effect of CASC9 on DOX-resistant breast cancer cells was investigated for the first time in the present study. Additionally, the potential mechanisms of CASC9 in breast cancer drug-resistant cells were evaluated by investigating the interactions between CASC9 and multidrug resistance 1 (MDR1). The present study aimed to provide some theoretical basis for the underlying mechanism of DOX-resistant breast cancer.

\section{Materials and methods}

Tissue collection. Paired breast cancer and adjacent normal breast tissues were obtained from 48 female patients (age $50 \pm 11$ years) undergoing surgical breast cancer resection between January 2012 and December 2013 at The First Affiliated Hospital, University of South China (Hengyang, China). The clinicopathological data of patients are presented in Table I. The patients did not receive local or systemic treatment prior to surgery. All of the resected tissues were stored at $-80^{\circ} \mathrm{C}$ until total RNA extraction. The pathological stage, grade, nodal status and estrogen receptor status of samples were appraised by an experienced pathologist. American Joint Committee on Cancer stages were used to characterize the stages of patient samples. The experiments were approved by the Research Ethics Committee of the First Affiliated Hospital, University of South China; all patients provided written informed consent.

Cell lines and cell culture. The human breast adenocarcinoma MCF-7 (HTB-22 ${ }^{\mathrm{TM}}$ ) and MCF-7/doxorubicin (DOX) cell lines were cultured using Iscove's modified Dulbecco's medium (Sigma-Aldrich; Merck KGaA, Darmstadt, Germany) containing $40 \mathrm{Ag} / \mathrm{ml}$ gentamicin and $10 \%$ newborn calf serum (HyClone; GE Healthcare Life Sciences, Logan, UT, USA) at $37^{\circ} \mathrm{C}$ in an atmosphere with $5 \% \mathrm{CO}_{2}$. MDA-MB-231 (HTB-26 ${ }^{\mathrm{TM}}$ ), MDA-MB-157 (HTB-24 ${ }^{\mathrm{TM}}$ ), and MDA-MB-468 (HTB-132 ${ }^{\mathrm{TM}}$ ) human breast cancer cell lines were maintained in Dulbecco's modified Eagle's medium (DMEM; Sigma-Aldrich; Merck KGaA) supplemented with $10 \%$ fetal bovine serum (FBS; Gibco; Thermo Fisher Scientific, Inc., Waltham, MA, USA), $100 \mathrm{U} / \mathrm{ml}$ penicillin, and $100 \mu \mathrm{g} / \mathrm{ml}$ streptomycin. The normal human mammary epithelial cell line (MCF10A; CRL-10317 ${ }^{\mathrm{TM}}$ ) was cultured in DMEM. All cell lines were purchased from the American Type Culture Collection (Manassas, VA, USA). The drug-resistant variant (MCF-7/DOX) of the MCF-7 cell line was induced by stepwise selection following prolonged ( $>6$ months) treatment of MCF-7 cells with increasing concentrations of DOX at a range of $(0.5-25 \mathrm{nmol} / \mathrm{l})$ in the medium (19). Following 6 months of culturing in the presence of DOX, the half-maximal inhibitory concentrations $\left(\mathrm{IC}_{50}\right)$ for the MCF-7/DOX and parental MCF-7 cells were 24 and $1 \mathrm{Amol} / 1 \mathrm{DOX}$, respectively. Cells were seeded at a density of $0.5 \times 10^{6}$ viable cells per $100-\mathrm{mm}$ plate, and the medium was replaced every other day for 6 days. Trypsinized cells were washed with PBS and frozen at $-80^{\circ} \mathrm{C}$ immediately until subsequent analyses.

Vector construction and transfection. The silencing vector pS-CASC9 and empty pSilencer [negative control (NC), termed si-NC] were purchased from Guangzhou FitGene Biotechnology Co., Ltd. (Guangdong, China), and MCF-7/DOX cells at a density of $1 \times 10^{5}$ were transfected using Lipofectamine ${ }^{\circledR}$ 3000 reagent (Invitrogen; Thermo Fisher Scientific, Inc.), according to the manufacturer's protocols, following $24 \mathrm{~h}$ of culturing. The cell lines that expressed the vectors stably were selected using $400 \mu \mathrm{g} / \mathrm{ml}$ Geneticin (Invitrogen; Thermo Fisher Scientific, Inc.) for 2 weeks. Knockdown was confirmed and measured by reverse transcription-quantitative polymerase chain reaction (RT-qPCR). Enhancer of zeste homolog 2 (EZH2), MDR1 and control siRNAs were synthesized by Shanghai GenePharma Co., Ltd. (Shanghai, China). The sequences were: Si-EZH2-1 sense, GUGUAUGAGUUU AGAGUCATT-3'; si-MDR1 sense, CAGAAAGCUUAGUAC CAAAdTdT; and si-NC sense, UAACGACGCGACGACGUA AdTdT. Cells were seeded in a 6-well plate and cultured in antibiotic- and serum-free medium. At $60 \%$ confluence, cells were transfected using Oligofectamine ${ }^{\circledR} 2000$ (Invitrogen; Thermo Fisher Scientific, Inc.). Cells were refreshed with regular medium after 4-6 h of transfection and subjected to the measurement of knockdown efficiency using RT-qPCR.

To overexpress EZH2, the plasmid pcDNA-EZH2 was constructed by introducing a BamHI-EcoRI fragment containing the EZH2 cDNA into the same sites as pcDNA3.1. The pcDNA-EZH2 plasmid was transfected into MCF-7/DOX and the cell line stably expressing EZH2 was screened using G418.

RNA pull-down assay. The RNA pull-down assay was performed to identify the CASC9-binding candidate using a Pierce Magnetic RNA-Protein Pull-Down kit (Pierce; Thermo Fisher Scientific, Inc.). Briefly, the target RNA and antisense control RNA were labeled with biotin at the 3' end and purified using a Pierce RNA 3' End Desthiobiotinylation kit (Thermo Fisher Scientific, Inc.). A labeled RNA probe (50 pmol) was used to bind to streptavidin magnetic beads following incubation in 1X RNA Capture buffer for $30 \mathrm{~min}$ at room temperature. Subsequently, $200 \mu \mathrm{g}$ protein was incubated in protein-RNA binding buffer for $150 \mathrm{~min}$ at $4^{\circ} \mathrm{C}$ with agitation. The final RNA-magnetic bead-protein complexes were washed three times with wash buffer. Subsequently, $12 \mu$ l elution buffer was added to retrieve the pull-down protein products. The retrieved proteins were resolved in gradient $4-12 \%$ gel electrophoresis followed by mass spectrometry (MS) identification. In detail, proteins precipitated by RNA pull-down assays were subjected to NuPAGE 4-12\% BisTris gel electrophoresis and examined with silver stain using the Pierce Silver Stain kit (cat. no. 24612; Pierce; Thermo Fisher Scientific, Inc.). Specific bands only in the sense CASC9 lane were excised and analyzed by MS (GeneScience Pharmaceuticals Co., Ltd., Beijing, China).

Cell survival assay. Cell survival was determined using an MTT assay. Cells were plated in 96-well plates at $5 \times 10^{4}$ cells/well and $20 \mu \mathrm{l}$ of MTT solution $(5 \mathrm{mg} / \mathrm{ml}$; Sigma-Aldrich; Merck KGaA) was added to each well for $4 \mathrm{~h}$ of incubation. The MTT solution was then removed and $200 \mu \mathrm{l}$ dimethyl sulfoxide (Sigma-Aldrich; Merck KGaA) was added to dissolve the crystals. Optical density was measured at a wavelength of $490 \mathrm{~nm}$ using a microplate reader.

$R T-q P C R$. Total RNA was isolated using the RNAiso ${ }^{\mathrm{TM}}$ Plus kit (Takara Bio, Inc., Otsu, Japan), and $1 \mu \mathrm{g}$ total RNA was 
Table I. Clinicopathological data of patients.

\begin{tabular}{|c|c|c|c|c|}
\hline \multirow[b]{2}{*}{ Clinicopathological feature } & \multirow[b]{2}{*}{ No. patients } & \multicolumn{2}{|c|}{ Expression of CASC9 } & \multirow[b]{2}{*}{ P-value } \\
\hline & & Lower & Higher & \\
\hline Age, years & & & & 0.246 \\
\hline$\leq 50$ & 20 & 4 & 16 & \\
\hline$>50$ & 28 & 3 & 25 & \\
\hline Histological grade & & & & 0.023 \\
\hline Grade 1 and 2 & 33 & 10 & 23 & \\
\hline Grade 3 & 15 & 4 & 11 & \\
\hline Tumor size, $\mathrm{cm}$ & & & & 0.342 \\
\hline$\leq 2$ & 26 & 4 & 22 & \\
\hline$>2$ & 22 & 3 & 19 & \\
\hline Lymph node metastasis & & & & 0.017 \\
\hline Absent & 18 & 15 & 3 & \\
\hline Present & 30 & 8 & 22 & \\
\hline AJCC stage & & & & 0.188 \\
\hline I and II & 26 & 3 & 23 & \\
\hline III and IV & 22 & 4 & 18 & \\
\hline Lymphovascular invasion & & & & 0.034 \\
\hline Absent & 20 & 3 & 17 & \\
\hline Present & 28 & 6 & 22 & \\
\hline ER expression & & & & 0.026 \\
\hline Negative & 14 & 3 & 11 & \\
\hline Positive & 34 & 6 & 28 & \\
\hline
\end{tabular}

CASC9, cancer susceptibility candidate 9; ER, estrogen receptor; AJCC, American Joint Committee on Cancer.

reverse-transcribed at $70^{\circ} \mathrm{C}$ into first-strand cDNA using a Takara RNA PCR kit (AMV) v3.0 (Takara Biotechnology Co., Ltd., Dalian, China). The primer sequences used were as follows: Human EZH2 gene forward, 5'-GCCAGACTGGGA AGAAATCTG-3' and reverse, 5'-TGTGCTGGAAAATCC AAGTCA-3'; MDR1 gene forward, 5'-CCCATCATTGCAATA GCAGG-3' and reverse, 5'-GTTCAAACTTCTGCTCCTGA-3'; and $\beta$-actin (internal control) forward, 5'-ACCCCCACTGAA AAAGATGA-3' and reverse, 5'-ATCTTCAAA CCTCATGAT G-3'. Following heating to $94^{\circ} \mathrm{C}$ for $2 \mathrm{~min}$, the experimental reaction $(50 \mu \mathrm{l})$ was subjected to 32 cycles of $94^{\circ} \mathrm{C}$ for $30 \mathrm{sec}$, $61^{\circ} \mathrm{C}$ for $30 \mathrm{sec}$, and $72^{\circ} \mathrm{C}$ for $30 \mathrm{sec}$. The expression levels were calculated using the $2^{-\Delta \Delta \mathrm{Cq}}$ method (20).

Apoptosis analysis. Cells were trypsinized, and washed with cold PBS, and subsequently suspended in PBS. The apoptotic cells were detected by Annexin $\mathrm{V}$ and propidium iodide (PI) dual labeling using an Annexin V-fluorescein isothiocyanate (FITC) kit (Beijing Biosea Biotechnology Co., Ltd., Beijing, China), according to the manufacturer's protocols. A total of $24 \mathrm{~h}$ following cell transfection, breast cancer MCF-7 (HTB-22 ${ }^{\mathrm{TM}}$ ) and MCF-7/DOX cells were cultured in serum-free DMEM. The cells were harvested and washed three times using PBS buffer ( $\mathrm{pH} 7.4)$, and then resuspended in staining buffer. Subsequently, $5 \mu \mathrm{l}$ Annexin V-FITC and $5 \mu \mathrm{l}$ PI was added into the cells and incubated at room temperature for $10 \mathrm{~min}$. The mixtures were analyzed using a FACScan flow cytometer (BD Biosciences, Franklin Lakes, NJ, USA). Annexin V-positive and PI-negative cells were considered to be apoptotic cells. The apoptotic cells were analyzed using CellQuest software (version 3.0; BD Biosciences).

Cell migration and invasion assays. For the migration assay, at $48 \mathrm{~h}$ post-transfection, $5 \times 10^{4}$ cells in serum-free medium were placed into the upper chamber of an insert (8-mm pore size; EMD Millipore, Billerica, MA, USA). For the invasion assay, $1 \times 10^{5}$ cells in serum-free medium were placed into the upper chamber of an insert coated with Matrigel. The lower chamber was filled with medium containing 10\% FBS. Following incubation at $37^{\circ} \mathrm{C}$ for $24 \mathrm{~h}$, the cells remaining on the upper membrane were removed with cotton wool; cells migrating or invading through the membrane were stained with methanol and $0.1 \%$ crystal violet at room temperature, and imaged and counted using an IX71 inverted microscope at x400 magnification (Olympus Corporation, Tokyo, Japan).

Western blot analysis. Cells were washed twice with ice-cold PBS, and lysed using $2 \mathrm{ml}$ lysis buffer (radioimmunoprecipitation assay buffer; Sangon Biotech Co., Ltd., Shanghai, China). The supernatant was collected following centrifugation at $6,000 \times \mathrm{g}$ for $15 \mathrm{~min}$ at $4^{\circ} \mathrm{C}$ and cell lysates were matched for protein concentration using a bicinchoninic acid protein 
assay kit (Pierce; Thermo Fisher Scientific, Inc.). Protein samples (50 $\mu \mathrm{g}$ per lane) were loaded onto a 10\% SDS-PAGE gel and transferred onto nitrocellulose membranes, and subsequently blocked in $5 \%$ non-fat milk at $4{ }^{\circ} \mathrm{C}$ overnight. The membrane was incubated with primary antibodies with dilution 1:1,000 (Bcl2, cat. no. ab32124; Bax, cat. no. ab32503; caspase-3, cat. no. ab32150; caspase-9, cat. no. ab138412; and EZH2, cat. no. ab191250; all from Abcam, Cambridge, UK) for $2 \mathrm{~h}$ at room temperature, and subsequently incubated with HRP-conjugated secondary antibodies (cat. no. ab6721; dilution 1:5,000; Abcam) for $1 \mathrm{~h}$ at room temperature. The bands were visualized using enhanced chemiluminescence substrates (Thermo Fisher Scientific, Inc.).

Statistical analysis. All experiments were independently repeated three times. Values are presented as the mean \pm standard deviation. SPSS 16.0 software (SPSS, Inc., Chicago, IL, USA) was used for statistical analysis. The analysis of multiple groups was performed with one-way analysis of variance with Dunnett's post hoc test. $\mathrm{P}<0.05$ was considered to indicate a statistically significant difference.

\section{Results}

CASC9 is upregulated in breast cancer tissues and breast cancer drug-resistant cell lines. To investigate the effects of CASC9 on breast cancer, the expression of CASC9 in breast cancer tissues and cell lines was detected. The results of the RT-qPCR analysis revealed that CASC9 was significantly upregulated in breast cancer tissues (Fig. 1A) and cell lines (MCF-7, MCF-7/DOX, MDA-MB-231, MDA-MB-157 and MDA-MB-468) compared with in adjacent normal tissues and normal human mammary epithelial cells MCF10A (Fig. 1B and 1C). These results suggested that CASC9 may serve an important role in the pathogenesis and mechanism of drug-resistance in breast cancer.

CASC9 knockdown inhibits the growth, metastasis and chemoresistance of drug-resistant breast cancer cells. To study whether abnormal expression of CASC 9 affected the growth and metastasis of drug-resistant breast cancer cells, MCF-7/DOX cells that stably expressed a CASC9 silencing vector were established. The knockdown of CASC9 was confirmed by RT-qPCR (Fig. 2A). Subsequently, the cell viability, apoptosis, migration and invasion of MCF-7/DOX cells following CASC9 knockdown was investigated. As presented in Fig. 2B, CASC9 knockdown significantly inhibited the cell viability of MCF-7/DOX cells from $72 \mathrm{~h}$ following cell culture. The results of the flow cytometry demonstrated that CASC 9 knockdown significantly increased the apoptosis of MCF-7/DOX cells (Fig. 2C). The expression levels of apoptosis-associated proteins [apoptosis regulator Bcl-2 (Bcl-2), apoptosis regulator BAX (Bax), caspase-3 and caspase-9] are presented in Fig. 2D. The expression levels of Bcl-2, pro-caspase-3 and pro-caspase-9 decreased significantly following CASC9 knockdown, whereas Bax, cleaved-caspase- 3 and cleaved-caspase- 9 increased markedly. A Transwell assay revealed that CASC9 knockdown in MCF-7/DOX cells significantly impeded cell migration and invasion (Fig. 2E and 2F).
In addition, the $\mathrm{IC}_{50}$ of DOX in MCF-7/DOX cells following CASC9 knockdown was detected. CASC9 knockdown in MCF-7/DOX cells significantly decreased the $\mathrm{IC}_{50}$ of DOX (Fig. 2G).

Identification of EZH2 as a CASC9 binding protein. To further understand the underlying mechanism of CASC9 in breast cancer cells, a protein that potentially interacts with CASC9 was investigated using RNA pull-down assays followed by MS (Fig. 3A). EZH2 is a transcriptional repressor, which has been reported to be a marker of aggressive breast cancer (21); EZH2 may interact with CASC9. Therefore, CASC9 was conjugated to D-Biotin, and the associations between CASC9 and EZH2 were analyzed using recombinant $\mathrm{EZH} 2$ via the in vitro streptavidin-binding assay. As a result, EZH2 was demonstrated to be a binding protein of CASC9 (Fig. 3B). To investigate whether CASC9 affected the stability of EZH2, MCF-7 cells were transfected with Flag-CASC9 and the expression of EZH2 was detected. As presented in Fig. 3C, overexpressed CASC9 may significantly increase the protein expression of EZH2. Furthermore, the $\mathrm{IC}_{50}$ of DOX in MCF-7/DOX cells following CASC9 knockdown and EZH2 overexpression was determined. CASC9 knockdown significantly decreased the $\mathrm{IC}_{50}$ of DOX (Fig. 3D). Conversely, EZH2 overexpression reversed the inhibitory effect of CASC9 knockdown on the $\mathrm{IC}_{50}$ of DOX, which suggested that CASC9 promoted DOX-resistance by binding with EZH2.

EZH2 expression is increased in breast cancer tissues and breast cancer drug-resistant cell lines. The expression levels of EZH2 in breast cancer tissues and cells was detected. As presented in Fig. 3E, EZH2 expression levels were significantly higher in breast cancer tissues compared with in the adjacent normal tissues. Additionally, EZH2 expression within the breast cancer cells (MDA-MB-231, MDA-MB-157 and MDA-MB-468) and MCF-7/DOX cells was significantly higher compared with in MCF10A cells. Additionally, compared with MCF-7, MDA-MB-231, MDA-MB-157 and MDA-MB-468, EZH2 expression in MCF-7/DOX was significantly increased (Fig. 3F).

Effects of EZH2 siRNA on the cell growth, metastasis and chemoresistance of drug-resistant breast cancer cells. To investigate whether the abnormal expression of EZH2 affected the growth and metastasis of drug-resistant breast cancer cells, MCF-7/DOX cells were transfected with EZH2 siRNA. The expression levels of EZH2 decreased significantly post-transfection (Fig. 4A and B). Cell viability, apoptosis, migration and invasion following EZH2 silencing were detected. As Presented in Fig. 4C, EZH2 silencing significantly inhibited the viability of MCF-7/DOX cells from $72 \mathrm{~h}$ following cell culture. Flow cytometry revealed that EZH2 silencing significantly increased the apoptotic rate of MCF-7/DOX cells (Fig. 4D). Additionally, the expression levels of Bcl-2, pro-caspase-3 and pro-caspase-9 decreased markedly, while those of Bax, cleaved-caspase- 3 and cleaved-caspase- 9 increased markedly following EZH2 knockdown (Fig. 4E). The Transwell assay revealed that EZH2 silencing significantly inhibited cell migration and invasion (Fig. 4F and G). The $\mathrm{IC}_{50}$ of MCF-7/DOX cells to DOX following EZH2 knockdown were investigated; 



Figure 1. Expression of long noncoding RNA CASC9 in breast cancer tissue and cell lines. (A) Expression levels of CASC9 in breast cancer tissue. ${ }^{* *} \mathrm{P}<0.01$ vs. non-tumor tissue. (B) MCF-7 and drug-resistant MCF-7/DOX breast cancer cells were detected via reverse transcription-quantitative polymerase chain reaction. ${ }^{* *} \mathrm{P}<0.01$ vs. MCF10A group; ${ }^{\# \# \#} \mathrm{P}<0.001$ vs. MCF-7 group $\mathrm{C} ;{ }^{++} \mathrm{P}<0.01$. (C) The relative mRNA expression of CASC9 in three variants of the MDA-MB cell line. ${ }^{* *} \mathrm{P}<0.01$ vs. MCF10A group. CASC9, cancer susceptibility candidate 9 ; DOX, doxorubicin.

A

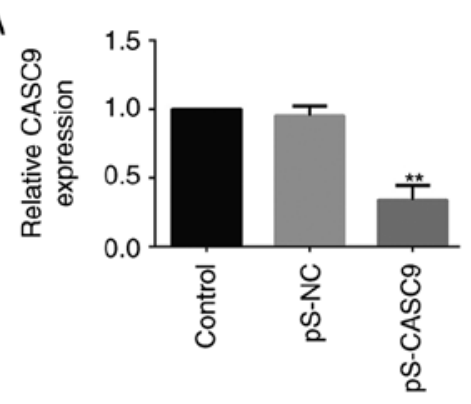

$\mathrm{B}$

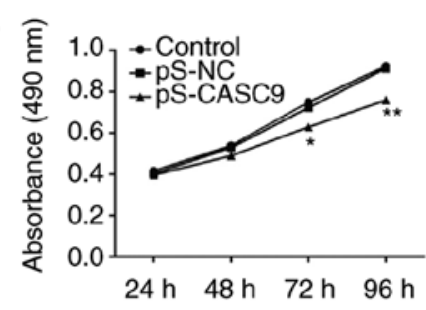

C

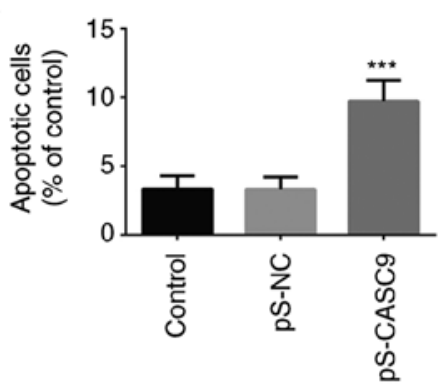

$\mathrm{D}$



$\mathrm{F}$

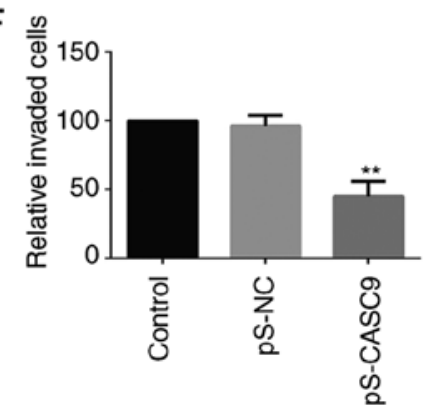

E

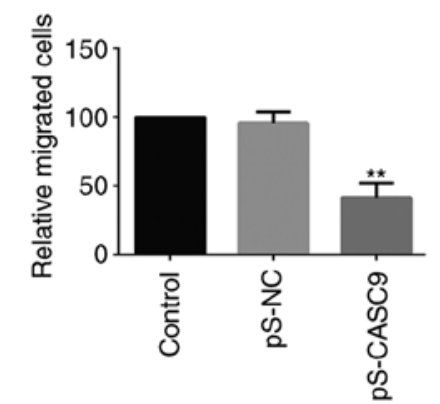

G

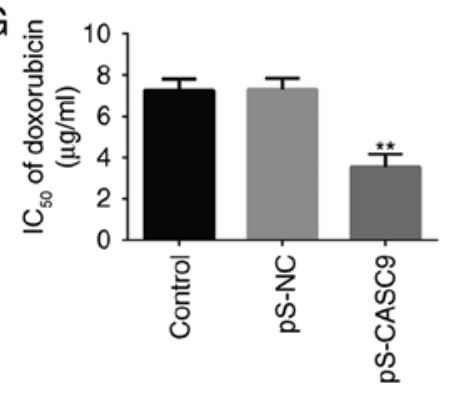

Figure 2. Effects of CASC9 on the growth and metastasis of drug-resistant breast cancer cells MCF-7/DOX. (A) Relative expression levels of CASC9 following cell transfection as confirmed by reverse transcription-quantitative polymerase chain reaction. Alterations in the (B) cell viability and (C) apoptosis of MCF-7/DOX cells following CASC9 knockdown. (D) Expression levels of apoptosis-associated proteins (Bcl-2, Bax, caspase-3 and caspase-9) in MCF-7/DOX following CASC9 knockdown. Alterations in the (E) cell migration and (F) invasion abilities of MCF-7/DOX cells following CASC9 knockdown. (G) Alterations in the $\mathrm{IC}_{50}$ of DOX in MCF-7/DOX cells following CASC9 knockdown. ${ }^{* *} \mathrm{P}<0.05,{ }^{* *} \mathrm{P}<0.01$ and ${ }^{* * * *} \mathrm{P}<0.001$ vs. the control group. Bcl-2, apoptosis regulator Bcl-2; Bax, apoptosis regulator BAX; CASC9, cancer susceptibility candidate 9; DOX, doxorubicin; IC $_{50}$, half-maximal inhibitory concentration.

EZH2 knockdown in MCF-7/DOX cells decreased the $\mathrm{IC}_{50}$ of DOX significantly (Fig. 4H).

Effects of EZH2 siRNA on the expression of MDR1/ $P$-glycoprotein $(P$-gp). Previously, it was suggested that the multidrug resistance of tumors is associated with the MDR1 gene (22). Therefore, the expression levels of the MDR1 gene and its encoded protein P-gp in drug-resistant breast cancer cells were detected. The expression levels of MDR1/P-gp in MCF-7/DOX cells increased significantly compared with in 
A



B

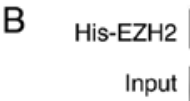

$\begin{array}{rll}\text { Biotin-CASC9 } & - & + \\ \text { His-EZH2 } & + & + \\ \text { Streptavidin beads } & + & +\end{array}$

C EZH2 GAPDH

\section{Flag-CASC9}

HA-EZH2
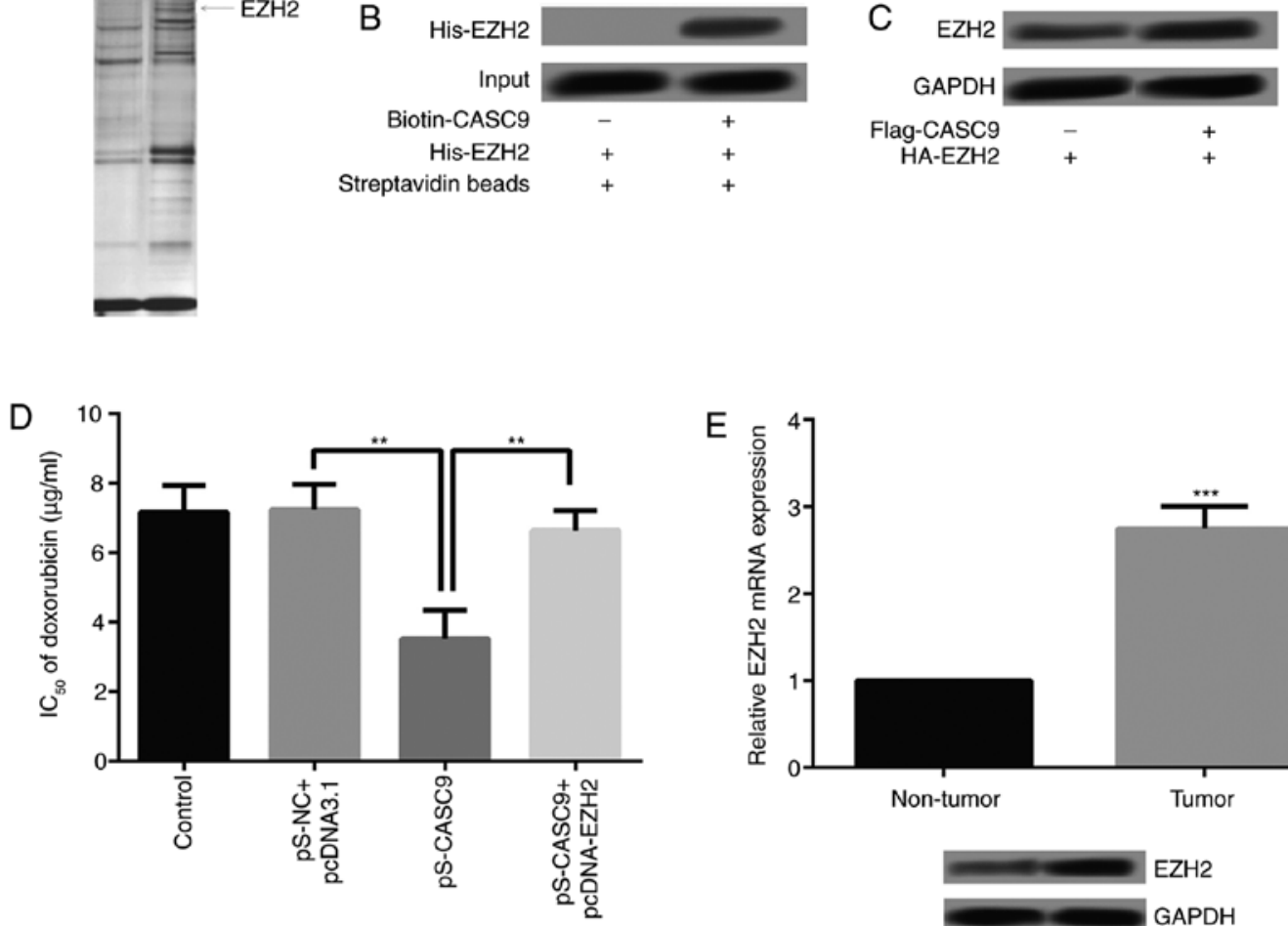

$\mathrm{E}$


Figure 3. Target gene expression of EZH2 and the correlation between EZH2 and CASC9. (A) EZH2 bound to CASC9 as detected by an RNA pull-down assay. (B-D) Overexpressed CASC9 significantly increased the protein expression of EZH2. (E) The expression levels of EZH2 in breast cancer tissue detected by RT-qPCR. (F) Expression level of EZH2 in breast cells MCF-7, MDA-MB-231, MDA-MB-157 and MDA-MB-468, and drug-resistant breast cancer cells MCF-7/DOX detected by RT-qPCR. ${ }^{* *} \mathrm{P}<0.05$ and ${ }^{* * * *} \mathrm{P}<0.001$ vs. the control group; ${ }^{*} \mathrm{P}<0.05$ vs. MCF-7/DOX. CASC9, cancer susceptibility candidate 9 ; DOX, doxorubicin; EZH2, enhancer of zeste homolog; NC, negative control; pS, vector; RT-qPCR, reverse transcription-quantitative polymerase chain reaction.

MCF-7, MDA-MB-231, MDA-MB-157 and MDA-MB-468 cells (Fig. 5A).

It has been reported that RNA interference-mediated EZH2 depletion may reduce MDR1 expression and sensitize multidrug-resistant tumor cells to chemotherapy (23-25). Considering the well-established role of EZH2 in regulating MDR1/P-gp expression in certain cancer types, the effects of EZH2 depletion on MDR1/P-gp levels were detected in drug-resistant breast cancer cells. As presented in Fig. 5B, EZH2 silencing resulted in suppressed MDR1/P-gp expression compared with in cells transfected with control or siRNA-NC.

Effects of CASC9 on the cell growth, metastasis and chemoresistance of drug-resistant breast cancer cells via regulation of MDRI expression. To further explore the mechanisms underlying the influence of CASC9 on the cell 

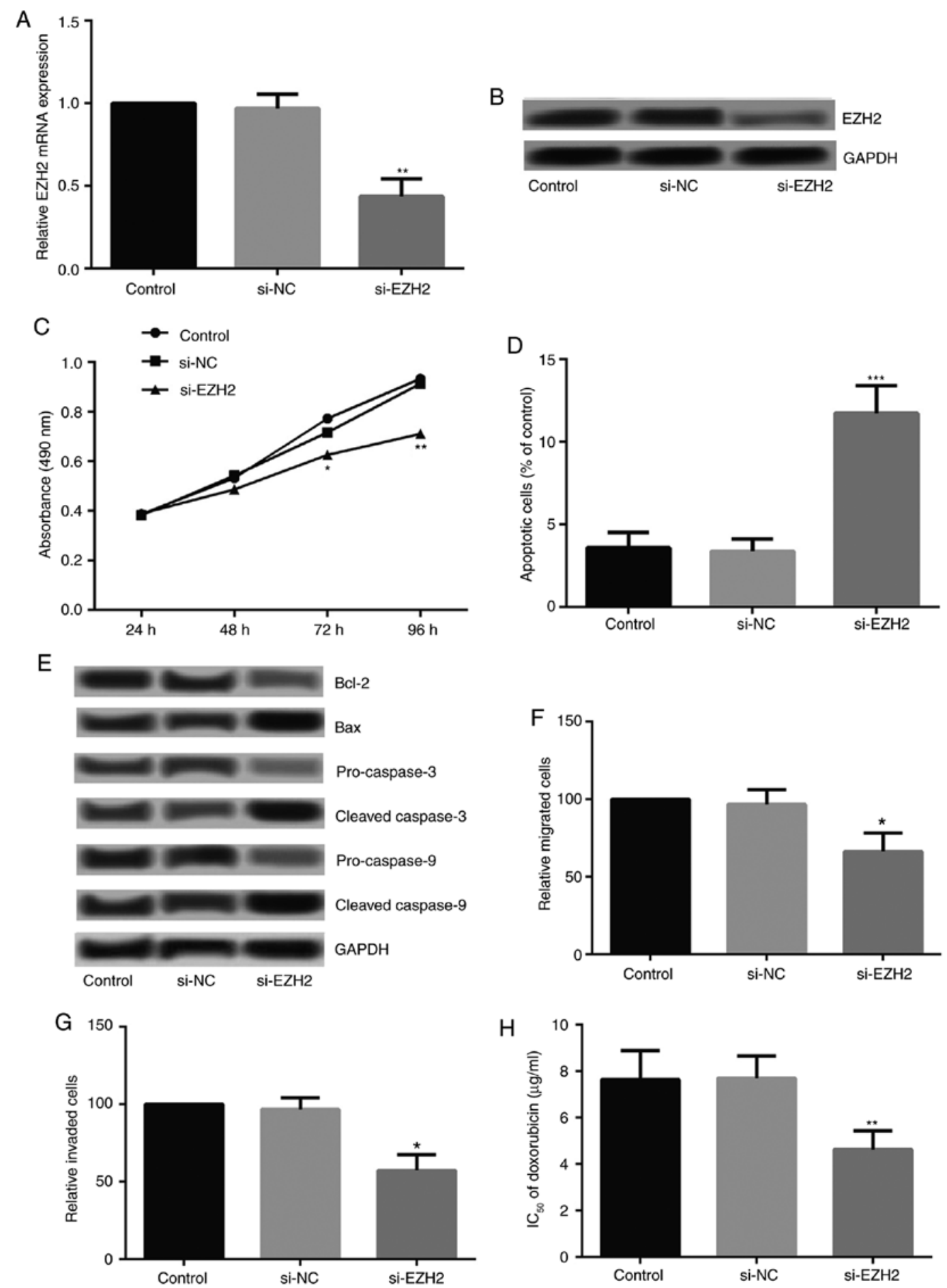

Figure 4. Effects of EZH2 on the growth and metastasis of drug-resistant breast cancer cells MCF-7/DOX. Relative expression levels of EZH2 following cell transfection, confirmed by (A) reverse transcription-quantitative polymerase chain reaction and (B) western blotting. Alterations in the (C) cell viability and (D) apoptosis of MCF-7/DOX following EZH2 silencing. (E) Expression levels of apoptosis-associated proteins (Bcl-2, Bax, caspase-3 and caspase-9) in MCF-7/DOX following EZH2 silencing. Alterations in the (F) cell migration and (G) invasion abilities of MCF-7/DOX cells following EZH2 silencing. $(\mathrm{H})$ Alterations in the $\mathrm{IC}_{50}$ of DOX in MCF-7/DOX cells following EZH2 silencing. ${ }^{*} \mathrm{P}<0.05,{ }^{* *} \mathrm{P}<0.01$ and ${ }^{* * *} \mathrm{P}<0.001$ vs. the control group. Bcl-2, apoptosis regulator Bcl-2; Bax, apoptosis regulator BAX; DOX, doxorubicin; EZH2, enhancer of zeste homolog; NC, negative control; si, small interfering RNA.

growth, metastasis and chemoresistance of MCF-7/DOX cells, MDR1 was silenced by transfecting MCF-7/DOX cells with MDR1 siRNA. The knockdown effect was confirmed by measuring the mRNA and protein expression levels (Fig. 5C). Subsequent analysis demonstrated that compared with the CASC9 knockdown and control groups, cell viability in the CASC9 knockdown + si-MDR1 group decreased significantly
(Fig. 5D). Additionally, CASC9 knockdown + si-MDR1 further promoted apoptosis in MCF-7/DOX cells (Fig. 5E), and affected the expression of apoptosis-associated proteins (Fig. 5F). Furthermore, significant reductions in the migration and invasion of MCF-7/DOX cells were detected in the CASC9 knockdown + si-MDR1 group compared with the CASC9 knockdown and control groups (Fig. 5G and 5H). 

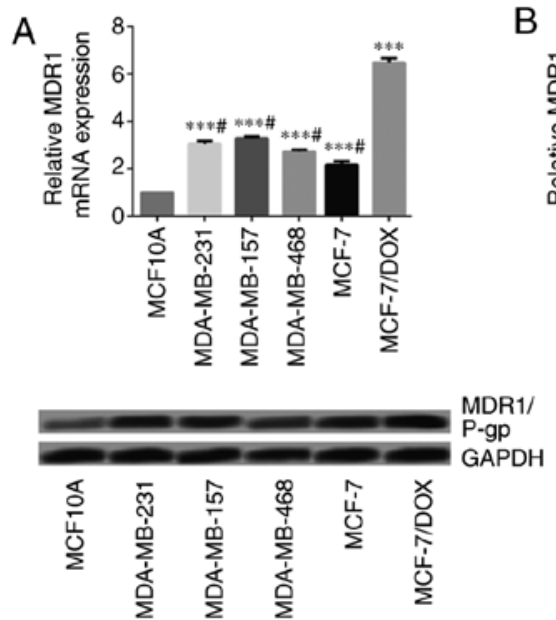
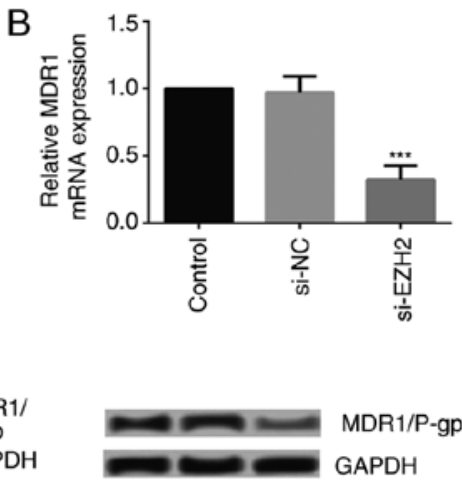

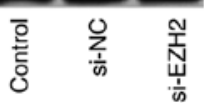

D

$\rightarrow$ Control

- pS-NC+si-NC

+ pS-CASC

* si-MDR1

$\rightarrow$ pS-CASC9+si-MDR1

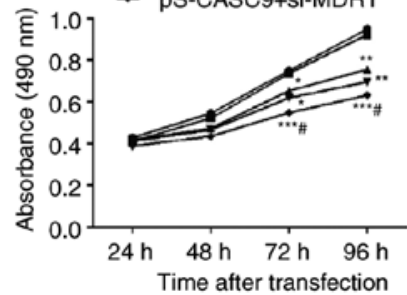

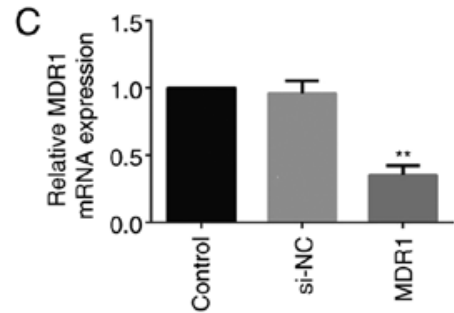



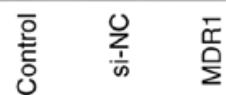

E


F
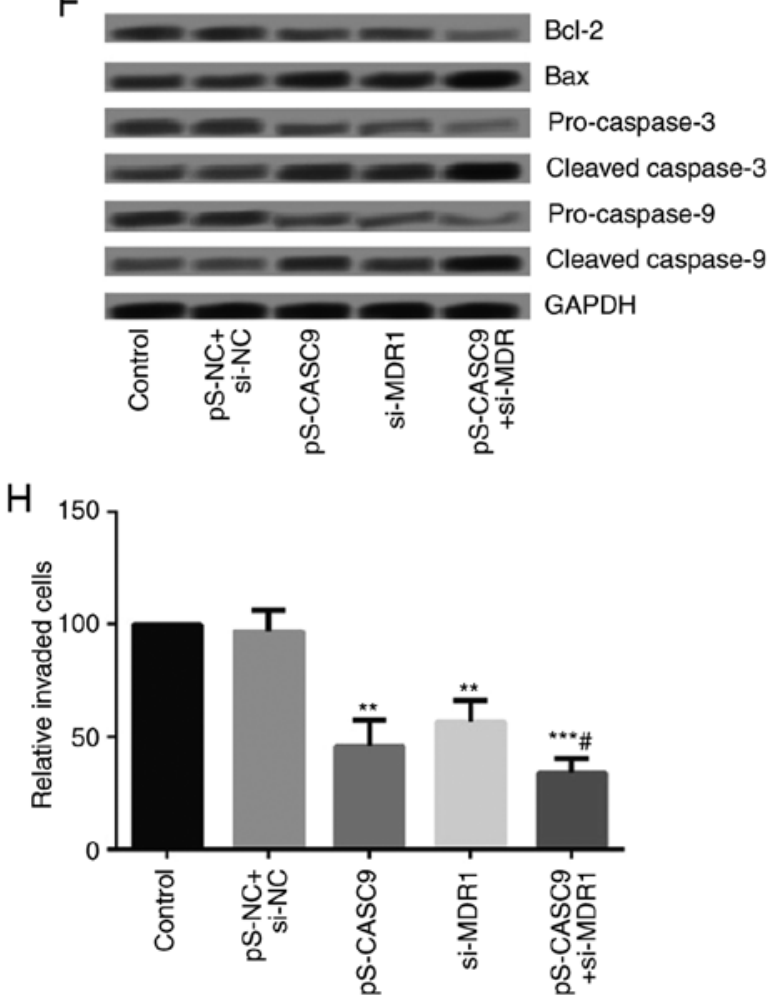

Figure 5. Effects of EZH2 on the growth and metastasis of drug-resistant breast cancer cells MCF-7/DOX. (A) Relative expression levels of MDR1/P-gp in MCF-7, MDA-MB-231, MDA-MB-157, and MDA-MB-468 and MCF-7/DOX cells detected by RT-qPCR and western blotting. ${ }^{* * *} \mathrm{P}<0.001 \mathrm{vs.} \mathrm{MCF10A}$ group; "P<0.05 vs. MCF-7/DOX group. (B) Relative expression levels of MDR1/P-gp following EZH2 silencing as detected by RT-qPCR and western blotting. ${ }^{* * * *} \mathrm{P}<0.001$ vs. control group. (C) Relative expression levels of MDR1 following cell transfection as confirmed by RT-qPCR and western blotting. ${ }^{* * *} \mathrm{P}<0.01 \mathrm{vs.} \mathrm{con}-$ trol group. Alterations in the (D) cell viability of MCF-7/DOX following CASC9 and MDR1 knockdown. ${ }^{*} \mathrm{P}<0.05,{ }^{* *} \mathrm{P}<0.01$ and ${ }^{* * * *} \mathrm{P}<0.001$ vs. pS-NC + si-NC group; ${ }^{~} \mathrm{P}<0.05$ vs. pS-CASC9 group. (E) Apoptosis of MCF-7/DOX following CASC9 and MDR1 knockdown. ${ }^{* *} \mathrm{P}<0.01$ and ${ }^{* * * *} \mathrm{P}<0.001$ vs. pS-NC + si-NC group; " $\mathrm{P}<0.05$ vs. pS-CASC9 group. (F) Expression levels of apoptosis-associated proteins (Bcl-2, Bax, caspase-3 and caspase-9) in MCF-7/DOX following CASC9 and MDR1 knockdown. Alterations in the $(\mathrm{G})$ cell migration and $(\mathrm{H})$ invasion abilities of MCF-7/DOX cells following CASC9 and MDR1 knockdown. ${ }^{*} \mathrm{P}<0.05,{ }^{* *} \mathrm{P}<0.01$ and ${ }^{* * * *} \mathrm{P}<0.001$ vs. pS-NC + si-NC group; ${ }^{*} \mathrm{P}<0.05$ vs. pS-CASC9 group. Bcl-2, apoptosis regulator Bcl-2; Bax, apoptosis regulator BAX; DOX, doxorubicin; EZH2, enhancer of zeste homolog; NC, negative control; P-gp, P-glycoprotein; pS, vector; RT-qPCR, reverse transcription-quantitative polymerase chain reaction; si, small interfering RNA; CASC9, cancer susceptibility candidate 9; MDR1, multidrug resistance protein 1. 


\section{Discussion}

The results of the present study revealed that CASC9 was upregulated in breast cancer tissues and cell lines, in addition to drug-resistant breast cancer cells. CASC9 knockdown inhibited the growth and metastasis of drug-resistant breast cancer cells, and decreased the $\mathrm{IC}_{50}$ of DOX in MCF-7/DOX cells. In addition, further study indicated that CASC 9 bound to EZH2 which regulated the MDR1 gene, a crucial factor in drug resistance. These interactions may serve an important role in the development of breast cancer cell resistance to chemotherapeutic drugs.

CASC9 was originally detected in esophageal squamous cell carcinoma, and a higher expression level of CASC9 was correlated with poor differentiation in esophageal squamous cell carcinoma $(16,26)$. A recent study demonstrated that CASC9 was frequently overexpressed in gastric cancer. Furthermore, it may promote cell growth and chemoresistance to adriamycin in gastric cancer (27). To the best of our knowledge, the present was the first to suggest the upregulation of CASC9 in breast cancer. Furthermore, CASC9 knockdown inhibited cell growth and metastasis, and reduced the chemoresistance of drug-resistant breast cancer cells to DOX. These findings were consistent with the previously mentioned studies, and may indicate the important role of CASC9 in human cancer.

EZH2, a transcriptional repressor, has been suggested to serve a critical role in the tumorigenic process as it has been revealed to be overexpressed in a number of malignancies, including lymphoma (28), prostate cancer (29) and bladder cancer (30). Importantly, numerous studies have confirmed that increased expression of EZH2 is associated with a high histological grade and worse survival in breast cancer, suggesting its promising role as a prognostic biomarker in aggressive breast cancer $(21,31)$. In the present study, RNA pull-down assays revealed that EZH2 potentially interacted with CASC9. Overexpressed CASC9 significantly increased the protein expression of EZH2. In addition, EZH2 silencing inhibited cell growth and metastasis, and reduced the $\mathrm{IC}_{50}$ of DOX in MCF-7/DOX cells. Therefore, CASC9 may serve roles in breast cancer progression and chemoresistance to DOX by binding to EZH2 .

Studies have reported that the multidrug resistance of a tumor may be caused by MDR1/P-gp, which is encoded by the human MDR1 gene. MDR1/P-gp is an integral membrane protein, whose function is the energy-dependent export of substances from the inside of cells and from membranes to the outside $(32,33)$. MDR 1/P-gp is considered to render tumor cells resistant to chemotherapy via the effective elimination of these agents from cancer cells (34). Notably, numerous studies have reported that the silencing of EZH2 may lead to decreases in MDR1 expression $(23,24)$. The results of the present study revealed that EZH2 silencing suppressed the expression of MDR1/P-gp in MCF-7/DOX cells, suggesting that EZH2 may be involved in the transcriptional regulation of MDR1, consistent with the aforementioned studies.

In conclusion, the present study demonstrated the oncogenic role of CASC9 in breast cancer and drug-resistant breast cancer cells by binding to EZH2 and regulating the MDR 1 gene. These findings indicated that the modulation of CASC9 expression may be a promising target in therapy of breast cancer and drug-resistant breast cancer.

\section{Acknowledgements}

Not applicable.

\section{Funding}

The present study was supported by the Natural Science Foundation of Hunan Province (grant no. 2015JJ2119).

\section{Availability of data and materials}

The data that support the findings of this study are available from the First Affiliated Hospital of University of South China (Hengyang, China) but restrictions apply to the availability of these data, which were used under license for the current study, and so are not publicly available. Data are however available from the authors upon reasonable request and with permission of the First Affiliated Hospital of University of South China.

\section{Authors' contributions}

BJ wrote the manuscript and conducted the MTT assay, RNA pull-down assay, western blotting and RT-qPCR. YL designed the study and provided the foundation of the study. XQ, HZ and YT performed the cellular apoptosis analysis. QF and YJ contributed to the data analysis. ML and XW helped to collect data.

\section{Ethics approval and consent to participate}

The experiments were approved by the Research Ethics Committee of the First Affiliated Hospital, University of South China (Hengyang, China); all patients provided written informed consent.

\section{Patient consent for publication}

All patients provided written informed consent.

\section{Competing interests}

The authors declare that they have no competing interests.

\section{References}

1. Ferlay J, Héry C, Autier P and Sankaranarayanan R: Global burden of breast cancer. In: Breast cancer epidemiology. Springer, pp1-19, 2010.

2. Riaz M, van Jaarsveld MT, Hollestelle A, Prager-van der Smissen WJ, Heine AA, Boersma AW, Liu J, Helmijr J, Ozturk B, Smid M, et al: miRNA expression profiling of 51 human breast cancer cell lines reveals subtype and driver mutation-specific miRNAs. Breast Cancer Res 15: R33, 2013.

3. World Health Organization, World Cancer Report 2014.

4. Eifel P, Axelson JA, Costa J, Crowley J, Curran WJ Jr, Deshler A, Fulton S, Hendricks CB, Kemeny M, Kornblith AB, et al: National institutes of health consensus development conference statement: Adjuvant therapy for breast cancer, november 1-3, 2000. J Natl Cancer Inst 93: 979-989, 2001.

5. Blum RH and Carter SK: Adriamycin. A new anticancer drug with significant clinical activity. Ann Intern Med 80: 249-259, 1974.

6. Chen JS, Agarwal N and Mehta K: Multidrug-resistant MCF-7 breast cancer cells contain deficient intracellular calcium pools. Breast Cancer Res Treat 71: 237-247, 2002. 
7. Herman J, Mangala L and Mehta K: Implications of increased tissue transglutaminase (TG2) expression in drug-resistant breast cancer (MCF-7) cells. Oncogene 25: 3049-3058, 2006.

8. Roberti A, La Sala DL and Cinti C: Multiple genetic and epigenetic interacting mechanisms contribute to clonally selection of drug-resistant tumors: Current views and new therapeutic prospective. J Cell Physiol 207: 571-581, 2006.

9. Duesberg P, Li R, Sachs R, Fabarius A, Upender MB and Hehlmann R: Cancer drug resistance: The central role of the karyotype. Drug Resist Updat 10: 51-58, 2007.

10. Fojo T: Multiple paths to a drug resistance phenotype: Mutations, translocations, deletions and amplification of coding genes or promoter regions, epigenetic changes and microRNAs. Drug Resist Updat 10: 59-67, 2007.

11. Sevignani C, Calin GA, Siracusa LD and Croce CM: Mammalian microRNAs: A small world for fine-tuning gene expression. Mamm Genome 17: 189-202, 2006.

12. Bushati $\mathrm{N}$ and Cohen SM: microRNA functions. Annu Rev Cell Dev Biol 23: 175-205, 2007.

13. Spizzo R, Almeida MIe, Colombatti A and Calin GA: Long non-coding RNAs and cancer: A new frontier of translational research? Oncogene 31: 4577-4587, 2012.

14. Gibb EA, Brown CJ and Lam WL: The functional role of long non-coding RNA in human carcinomas. Mol Cancer 10: 38, 2011

15. Shore AN, Herschkowitz JI and Rosen JM: Noncoding RNAs involved in mammary gland development and tumorigenesis: There's a long way to go. J Mammary Gland Biol Neoplasia 17: 43-58, 2012.

16. Pan Z, Mao W, Bao Y, Zhang M, Su X and Xu X: The long noncoding RNA CASC9 regulates migration and invasion in esophageal cancer. Cancer Med 5: 2442-2447, 2016.

17. Song P, Jiang B, Liu Z, Ding J, Liu S and Guan W: A three-lncRNA expression signature associated with the prognosis of gastric cancer patients. Cancer Med 6: 1154-1164, 2017.

18. Su X, Li G and Liu W: The long noncoding RNA cancer susceptibility candidate 9 promotes nasopharyngeal carcinogenesis via stabilizing HIF1 $\alpha$. DNA Cell Biol 36: 394-400, 2017.

19. Chekhun VF, Lukyanova NY, Kovalchuk O, Tryndyak VP and Pogribny IP: Epigenetic profiling of multidrug-resistant human MCF-7 breast adenocarcinoma cells reveals novel hyper- and hypomethylated targets. Mol Cancer Ther 6: 1089-1098, 2007.

20. Livak KJ and Schmittgen TD: Analysis of relative gene expression data using real-time quantitative PCR and the 2(-Delta Delta C(T)) method. Methods 25: 402-408, 2001.

21. Kleer CG, Cao Q, Varambally S, Shen R, Ota I, Tomlins SA, Ghosh D, Sewalt RG, Otte AP, Hayes DF, et al: EZH2 is a marker of aggressive breast cancer and promotes neoplastic transformation of breast epithelial cells. Proc Natl Acad Sci USA 100: 11606-11611, 2003.

22. Hoffmeyer S, Burk O, Von Richter O, Arnold HP, Brockmöller J, Johne A, Cascorbi I, Gerloff T, Roots I, Eichelbaum M, et al: Functional polymorphisms of the human multidrug-resistance gene: Multiple sequence variations and correlation of one allele with P-glycoprotein expression and activity in vivo. Proc Natl Acad Sci USA 97: 3473-3478, 2000
23. Tang B, Zhang Y, Liang R, Gao Z, Sun D and Wang L: RNAi-mediated EZH2 depletion decreases MDR1 expression and sensitizes multidrug-resistant hepatocellular carcinoma cells to chemotherapy. Oncol Rep 29: 1037-1042, 2013.

24. Zhou W, Wang J, Man WY, Zhang QW and Xu WG: siRNA silencing EZH2 reverses cisplatin-resistance of human non-small cell lung and gastric cancer cells. Asian Pac J Cancer Prev 16: 2425-2430, 2015.

25. Zhang Y, Liu G, Lin C, Liao G and Tang B: Silencing the EZH2 gene by RNA interference reverses the drug resistance of human hepatic multidrug-resistant cancer cells to 5-Fu. Life Sci 92: 896-902, 2013.

26. Cao W, Wu W, Shi F, Chen X, Wu L, Yang K, Tian F, Zhu M, Chen $\mathrm{G}$, Wang $\mathrm{W}$, et al: Integrated analysis of long noncoding RNA and coding RNA expression in esophageal squamous cell carcinoma. Int J Genomics 2013: 480534, 2013.

27. Shang C, Sun L, Zhang J, Zhao B, Chen X, Xu H and Huang B: Silence of cancer susceptibility candidate 9 inhibits gastric cancer and reverses chemoresistance. Oncotarget 8: 15393-15398, 2017.

28. Visser HP, Gunster MJ, Kluin-Nelemans HC, Manders EM Raaphorst FM, Meijer CJ, Willemze R and Otte AP: The Polycomb group protein EZH2 is upregulated in proliferating, cultured human mantle cell lymphoma. Br J Haematol 112: 950-958, 2001.

29. Varambally S, Dhanasekaran SM, Zhou M, Barrette TR, Kumar-Sinha C, Sanda MG, Ghosh D, Pienta KJ, Sewalt RG, Otte AP, et al: The polycomb group protein EZH2 is involved in progression of prostate cancer. Nature 419: 624-629, 2002.

30. Raman JD, Mongan NP, Tickoo SK, Boorjian SA, Scherr DS and Gudas LJ: Increased expression of the polycomb group gene, EZH2, in transitional cell carcinoma of the bladder. Clin Cancer Res 11: 8570-8576, 2005

31. Collett K, Eide GE, Arnes J, Stefansson IM, Eide J, Braaten A, Aas T, Otte AP and Akslen LA: Expression of enhancer of zeste homologue 2 is significantly associated with increased tumor cell proliferation and is a marker of aggressive breast cancer. Clin Cancer Res 12: 1168-1174, 2006.

32. Callaghan R, Luk F and Bebawy M: Inhibition of the multidrug resistance P-glycoprotein: Time for a change of strategy? Drug Metab Dispos 42: 623-631, 2014

33. Rahbari NN, Mehrabi A, Mollberg NM, Müller SA, Koch M, Büchler MW and Weitz J: Hepatocellular carcinoma: Current management and perspectives for the future. Ann Surg 253: 453-469, 2011

34. Takara K, Sakaeda T and Okumura K: An update on overcoming MDR1-mediated multidrug resistance in cancer chemotherapy. Curr Pharm Des 12: 273-286, 2006. 